\title{
Interactive comment on "Haze pollution in winter and summer in Zibo, a heavily industrialized city neighboring the Jin-Jin-Ji area of China: source, formation, and implications" by Hui Li et al.
}

\section{Anonymous Referee \#2}

Received and published: 5 March 2018

General comments:

In this study, the authors presented the field measurements during two relatively short periods: Jan 15-25, 2015 and July 14-31, 2015 in a heavily industrialized city in China. The authors measured PM2.5, SO4, NO3, OC, EC, as well as gases NOx, SO2, CO and 03 . The authors also measured meteorological variables of wind speed, wind direction, pressure, temperature and relatively humidity. From these about 4 weeks' measurements, the authors tried to make conclusion of the source, formation of two smog events in winter, and one smog event in summer. The authors concluded that high concentration of precursor, photochemical activities, relative humidity and low mixed 
layer height favor the formation of smog. The authors also claimed that photochemistry and SO2 concentration had the biggest impact on sulfate; Relative humidity, NO2, and "Excess $\mathrm{NH} 4+$ " were the main influence factor for the formation of nitrate in winter, and some other conclusions.

Operational and routine measurements of $\mathrm{PM} 2.5, \mathrm{SO} 4, \mathrm{NO} 3, \mathrm{NH} 4, \mathrm{SO} 2, \mathrm{HNO} 3$, and O3 have been carried out around the world for many decades. For example, these measurements have been carried out routinely for over a hundred sites in US and Canada for over three decades. The mechanism of formation of SO4, NO3, and NH4 has been well understood, and has been implemented successfully in various air quality models. There is no need to explain these well-known knowledges again and again through another short-period campaign. I tried to find any "new" results or new methods from the paper, but I didn't find. Reading through the abstract, what I found is everything I have known through textbook. Even the well-known knowledge was not explained well by the authors using the short-period observations. In general, I didn't see any valuable new information was presented in the paper, some section was very poorly written, eg. Section 3.6 Regional contributions. I firmly suggest the paper be rejected.

Specific comments:

1. The role of meteorology in formation of smog.

The meteorological condition, coupled with emission, is the major factors in formation of smog events. In this paper, the authors provided pressure, relative humidity, wind speed and temperature. From the time series of these variables, I can't see the meteorological conditions during these four weeks. To analyze the meteorological conditions, the authors need to analyze the $500 \mathrm{hPa}$ and surface weather maps; need to provide the information of precipitation. The authors claimed that mixed layer height plays a role, but I don't know how the authors have the information of mixed layer height. Did the authors analyze the temperature profiles? Otherwise, how can you claim mixed 
layer height play a role. Even the authors did do a good job of analyzing the vertical temperature profile to show that the smog events are related to low mixed layer height, this is new knowledge. It is well known and obvious.

The authors provided the time series of pressure, how it is linked to air quality? How wind speed and temperature are linked to the observed pollution?

In general, I saw too many pre-assumption, instead of rigorous analysis in the paper.

2. Chemical composites of haze

Chemical composites of haze in Eastern China are mainly due to SO4, NO3, NH4 (these are due to the emission of $\mathrm{SO} 2$ and NOx) as well as organic matters. These are well known and have been shown in many previous publications.

\section{Diurnal variation}

Page 233-238 The authors linked the diurnal variation of air pollutants to air pressure, as a scientist of deep knowledge in meteorology, I don't see the justification. Page 2422461 . It is well known stable weather condition (low wind speed, under a high pressure system as indicated by weather map, not just pressure, inverse of low-level temperature profile) favors the formation of pollution event. This kind of common knowledge has been applied operationally in air quality forecast. In the analysis, the authors used too many "maybe" instead of rigorous reasoning.

It is common that due to solar radiation at the day time, the surface is heated, and the temperature profile changes. So usually during day time, the boundary height is higher than during night. This is especially true during summer time. But this is a general situation of meteorology. For a specific synoptic event, we can also have lower boundary level height during day time than during night.

Essentially, the authors used known knowledge to explain the diurnal variation, but I didn't see any new thought or idea by the authors. Also, in explanation, the authors used too much "maybe". 


\section{Sulfate}

Line 323-325: Here the authors looked at the correlations between EC and SO4. First, these two species are not too much linked. EC is from primary emission, and SO4 is mainly from oxidation of SO2. I didn't see a good correlation between them, even from the authors' figure 5. The reasoning in line 323-325 was totally not justified, and can be wrong. SO4 is oxidation from SO2. High value of SO4 is not necessarily associated with high value of SO2. Also we need to realize that the life time of SO4 and SO2 are very different.

Relative humidity can be associated with many different meteorological conditions. For example, high relative humidity can be more associated with cloudy days, which mean less solar radiation, or even raining days. It is not convincing and conclusive to derive something simply based the correlation between SO4 and EC under two categories of relative humidity.

In the Section, the authors talked about wet scavenging. But the authors didn't mention the precipitation during the period. Also Scavenging coefficient for SO4 and EC is also different.

Line 346-347: This is well known that due to the much more solar photons available during summer time, the atmospheric oxidation capacity is much higher than winder season. So much more percentage of $\mathrm{SO} 2$ is oxidized. There is really no need to demonstrate this again and again. Line 347-349: I don't see the justification.

In general, I didn't see any new knowledge from this section.

\section{Nitrate}

Nitrate can be in PM2.5 and PM10. For PM2.5, it is mainly formed from the reaction $\mathrm{NO} 3 /(\mathrm{NO} 3+\mathrm{HNO} 3)$. Unfortunately, in this study, the authors didn't measure HNO3. For $\mathrm{PM} 2.5$, nitrate should be dominantly associated with $\mathrm{NH} 4$, as $\mathrm{Ca}(\mathrm{NH} 4) 2$ or $\mathrm{NaNH} 4$ is 
mainly PM10.

Line 390-409: Again, the text in this part has too many "maybe"s. 1. HNO3 is formed through the oxidation of NOx. The authors didn't provide an explanation why relative humidity affects the oxidation of NOx.

Even $\mathrm{HNO} 3$ is formed in the air, whether it can be neutralized to nitrate depends on other factors: temperature, availability of $\mathrm{NH} 3$.

Line 410-437: Again, the authors used too many "maybe"s to explain the correlation between $\mathrm{NO} 3$ and NO2. The authors used "Excess NH4+" to explain the formation of nitrate. Actually, this so called "Excess NH4+" is the $\mathrm{NH} 4$ associated with nitrate. The "Excess NH4+" is not the reason, but the results. High concentration of PM2.5 nitrate is definitely associated with high "Excess NH4+", as NH4NO3 is the dominantly PM2.5 nitrate. The dependence of $\mathrm{NH} 4 \mathrm{NO} 3$ on temperature is well known. No need to demonstrate it again with another observation. The reasoning in this part is not acceptable. For oxidation of $\mathrm{NOx}$ to $\mathrm{HNO} 3$, and then formation of nitrate is a complicated process involving gas-phase oxidation by $\mathrm{O} 3$ and other photochemical reactions. The authors are suggested to consulate some recent publication, eg. Hauglustaine et al. (2014, ACP), Chen et al. (2016, ACP) and many others.

\section{Regional contributions}

What is fire map?

Do the authors want to emphasis that the smog events are due to low wind and stable meteorological conditions, and therefore it was mainly due to local emission; or the authors want to say that it was mainly due to transportation? The results presented in this Section were very confusing. In general, the results presented in this paper are very poorly analyzed. There are too many "maybe"s instead of solid and rigorous analysis. I didn't find any valuable new information. A solid and rigorous analysis the formation of a haze event requires a comprehensive air quality model simulation with proper emis-

Printer-friendly version

Discussion paper 
sion input, comprehensive meteorology, and comparison with observations. Instead the authors tried to use some very basic meteorological variable, mainly relative humidity to explain the formation of three smog events. I don't see any values that merit publication in ACP. I firmly suggest the paper be rejected.

\section{Summary and conclusions}

The authors are supposed to provide their main findings in this Section. If the authors feel they have produced some new findings, they should emphasize them here.

Interactive comment on Atmos. Chem. Phys. Discuss., https://doi.org/10.5194/acp-2018-83, 2018. 